\title{
Comunicación y conocimiento de los Servicios Sociales públicos. La difusión de los Servicios Sociales municipales en la Comunidad de Madrid
}

\author{
Communication and knowledge of public Social Services. \\ Dissemination of Municipal Social Services in the Comunidad \\ of Madrid
}

Adolfo BALTAR MORENO

Universidad Complutense de Madrid adolfobaltarmoreno@gmail.com

Recibido: 19/04/2013

Revisado: $13 / 05 / 2013$

Aceptado: 20/09/2013

Disponible on line: 27/06/2014

\section{Resumen}

Se presenta un análisis de la difusión de los servicios sociales de atención primaria de la Comunidad de Madrid a través de las instituciones que producen y gestionan estos servicios públicos, las Administraciones locales, en tanto que emisoras o principales productoras de proceso comunicativo.

Se parte de la hipótesis de que el modelo de difusión empleado por las Administraciones locales para promocionar sus servicios sociales es un factor que determina el nivel de conocimiento de este sistema de protección social e influye en la capacidad de acceso de la ciudadanía.

El trabajo realiza un diagnóstico a partir de la observación de los principales factores que determinan la comunicación de los servicios sociales municipales: estrategias, contenidos, publicidad, marketing, emplazamientos y problemáticas. Las conclusiones llevan a plantear la necesidad de llevar a cabo una difusión global de los servicios sociales públicos en tanto que sistema, suplementaria a la que realizan el resto de Administraciones públicas concernidas, lo que no sucede en la actualidad.

Palabras clave: Servicios Sociales, comunicación, publicidad, información, marketing de servicios públicos.

\begin{abstract}
The article analyses the publicizing of primary care social services in the Municipality (Comunidad) of Madrid through the local Administrations, the institutions that produce and manage these public services, as broadcasters or principal producers of this communication process.

It is based on the hypothesis that the model of publicity used by the local Administrations to promote their social services determines the level of public knowledge of this social protection system and influences people's access to the system.

The research presents a diagnosis based on the observation of the principal factors that determine the construction of the image of municipal social services: strategies, content, advertising, marketing, product placements and obstacles. The conclusions lead us to propose the need to carry out a global dissemination of information about public social services as a system, supplementary to the efforts of remaining public Administrations concerned, which is not happening at present.
\end{abstract}

Keywords: Social Services, communication, advertising, information, public services marketing.

Referencia normalizada: Baltar Moreno, A. (2014): «Comunicación y conocimiento de los Servicios Sociales públicos. La difusión de los Servicios Sociales municipales en la Comunidad de Madrid». Cuadernos de Trabajo Social, 27(1): 127-138.

Sumario: Introducción. 1. Materiales y métodos. 2. Resultados y discusión. 3. Conclusiones. 4. Referencias bibliográficas. 


\section{Introducción}

Desde sus inicios, los Servicios Sociales públicos, que nacen en España al albor de la etapa democrática, han pretendido superar una imagen fuertemente vinculada a la caridad y a la pobreza, basándose en una concepción universal de la atención social. Responden a las características de un sistema, pese a que no sea reconocido como tal de manera general, como sostiene Perdomo Molina (2009) y lo observan autores (Las Heras, 2002; Fantova, 2003; Subirats, 2007; Muñoz Luque, 2009). Así también lo indican Guillén y Vilà (2007):

Las normas autonómicas, ya desde sus inicios, explicitaron su deseo de ser sistema y lo denominaron de esta forma (...) con el fin de, según señalan algunas leyes en sus exposiciones de motivos, superar la situación anterior de heterogeneidad y dispersión orgánica y administrativa (p. 161).

Un sistema de protección social — según la clasificación clásica de Kahn y Kamerman, 1987 - con una base organizativa de marcado carácter local y con una legislación principalmente autonómica, sostenido en una serie de principios entre los que destacan el principio de igualdad, el de solidaridad y el de universalidad.

La necesidad de comunicar estos nuevos Servicios Sociales a la sociedad nace con la propia puesta en marcha del sistema. Los estudios que vinculan comunicación con Servicios Sociales públicos son escasos, pudiendo destacarse los trabajos de Grande Esteban, (1993, 2002), Charroalde (1996), García Herrero y Ramírez Navarro (2001) y Puig (2003, 2004). La trayectoria de la comunicación de los Servicios Sociales ha pasado, desde entonces, por varias etapas de desarrollo, acompañando a la propia evolución del sistema. Etapas que culminan con el último gran avance en materia social: la Ley de Promoción de la Autonomía personal y Atención a las personas en situación de Dependencia (2006).

En la actualidad, las informaciones de los medios de comunicación indican que el actual anteproyecto de reforma de la Ley de la Administración Local española contempla la obligación de las autonomías de hacerse cargo de los Servicios Sociales en el plazo de un año, a partir de su aprobación, inicialmente prevista para la primavera de 2013. Sin embargo, y hasta la fecha, han sido las administraciones locales las encargadas de la prestación y gestión del nivel de Atención Primaria de estos servicios, adquiriendo a su vez la responsabilidad de su difusión, y por lo tanto, desempeñando un importante papel en la construcción de la imagen del sistema.

La comunicación de los Servicios Sociales forma parte de la información de los servicios públicos y supone realizar estas acciones de interés general. Puig (2004) sostiene que la comunicación de un servicio público debe cumplir al menos tres funciones básicas: 1) dar a conocer el servicio, 2) facilitar su comprensión y su uso, y 3) motivar y movilizar al ciudadano (p. 141).

Sin embargo, los Servicios Sociales públicos continúan siendo el sistema de protección social menos conocido por la sociedad española: unos servicios desconocidos, confundidos en muchas ocasiones en los discursos mediáticos y políticos con otros servicios públicos, que también forman parte del gran sistema de protección social, y observados de manera prejuiciosa por muchos sectores de la sociedad.

No disponemos de cifras fiables y actualizadas sobre el porcentaje de la ciudadanía que conoce los Servicios Sociales públicos. Sin embargo, a partir de algunas investigaciones (Bueno Abad y Pérez Cosín, 2000, p. 53; Marcuello y Gutiérrez Resa, 2008, p. 191 ), podemos estimar una cifra entre el 50 y el 60 por ciento de la misma, si bien es un porcentaje que parece ir en aumento. Este nivel de desconocimiento sería especialmente elevado en los municipios urbanos grandes (Informe EDIS, 2000).

Al referirnos al conocimiento del sistema por la ciudadanía dos grandes cuestiones están presentes: la del conocimiento de los derechos sociales y la del acceso a los servicios públicos. A este respecto, Warin $(2010$, p. 2) al hablar de las personas que siendo perfectamente elegibles para poder acceder a una prestación social no acceden a ellas (los denominados non recours en Francia y non take up of social benefits en el Reino Unido), establece una tipología de este segmento de población. En ella, el autor identifica el desconocimiento de las modalidades de prestación como uno de los prin- 
cipales motivos para no acceder a una prestación social, por una falta de información sobre su existencia o su modo de acceso.

Desde esta perspectiva, el conocimiento de la oferta y del derecho de acceso a la misma es un elemento necesario para que la ciudadanía pueda recurrir a la atención social pública cuando lo necesite. «La accesibilidad o inaccesibilidad no es solo una cuestión de escalones y demás obstáculos físicos: la desinformación como barrera es tan excluyente o más, si cabe, que cualquier otra» (Charroalde, 1996, pp. 38-41).

Bajo el punto de vista de la comunicación, podemos distinguir varios elementos que facilitan o dificultan el conocimiento de los servicios públicos: el tipo de información que incluyen las acciones de comunicación; los públicos a los que se dirige; la visibilidad de los servicios en los espacios públicos; la manera de promocionar y publicitar los servicios o el esfuerzo económico y de tiempo que se dedica a la comunicación, etc. En definitiva, supone observar el proceso de elaboración y puesta en marcha de las actividades de difusión de los servicios que se transmite a la ciudadanía desde las administraciones públicas.

En nuestro estudio, partimos de la hipótesis de que el modelo que las administraciones públicas están empleando actualmente para difundir el sistema público de Servicios Sociales no corresponde al que merecería un sistema pretendidamente universal, ya que no está contribuyendo a aumentar su nivel de conocimiento entre la ciudadanía y, por lo tanto, su capacidad para acudir a él.

¿Cómo se organizan las administraciones públicas españolas para promocionar este sistema ante la ciudadanía? ¿Qué técnicas se emplean y cómo se hace? ¿Qué importancia se le concede a la comunicación en el proceso de prestación de los servicios? ¿Qué se comunica y por qué? ¿Qué problemas identifican las áreas de Servicios Sociales cundo difunden sus servicios?

La finalidad de este trabajo, por lo tanto, es establecer un diagnóstico del modelo actual de difusión de los Servicios Sociales que llevan a cabo las administraciones públicas para poder determinar su posible influencia en el nivel de conocimiento que la ciudadanía tiene del sistema.

\section{Materiales y métodos}

La investigación ha centrado la observación en las administraciones públicas españolas, en tanto que instituciones productoras de los servicios sociales públicos, y dentro de ellas, en la Administración Local, hasta la fecha encargada de producir y gestionar estos servicios, así como de difundirlos y promocionarlos, es decir los denominados Servicios Sociales municipales.

La selección de la técnica del cuestionario como herramienta de investigación ha tenido como finalidad obtener una visión de las características principales de la difusión de los Servicios Sociales municipales en una región y en un momento determinados, tal y como la ven los responsables de esos servicios de los diferentes ayuntamientos participantes en la investigación.

Para realizar esta observación se han recogido, a lo largo del primer semestre de 2010, una serie de datos de naturaleza cuantitativa, mediante un cuestionario de 23 ítems, dirigido a las áreas de Servicios Sociales de los municipios mayores de 20.000 habitantes de la región, regidos por la Ley 11/2003 de 27 de marzo, de Servicios Sociales de la Comunidad de Madrid.

La selección de las Administraciones locales como unidades de análisis del estudio ha venido determinada por la propia estructura organizativa del sistema. La Ley 7/1985, Reguladora de las Bases del Régimen Local (LBRL), determina que los municipios mayores de 20.000 habitantes sean los encargados de la prestación de los Servicios Sociales y de promoción y reinserción social, por tanto uno de los puntos clave de acceso al sistema.

El cuestionario se ha dirigido expresamente a las áreas de Servicios Sociales de los municipios de la muestra y, dentro de de ellas, a los responsables principales de la comunicación de estos servicios. De ahí que, según cada caso, han contestado al cuestionario diversos perfiles de profesionales (políticos, coordinadores, equipos técnicos). Las peticiones de participación en la investigación se hicieron a través de los registros municipales, buscando así una respuesta oficial de las instituciones (Tabla 1).

Para la elaboración del cuestionario, se realizaron previamente una serie de entrevistas 


\begin{tabular}{|l|l|}
\hline Universo & $\begin{array}{l}\text { Áreas de Servicios Sociales de los 31 municipios mayores de 20.000 } \\
\text { habitantes de la Comunidad de Madrid }\end{array}$ \\
\hline Muestra & $\begin{array}{l}\text { 20 áreas de Servicios Sociales (a través de sus concejales, directores } \\
\text { y coordinadores de área) }\end{array}$ \\
\hline $\begin{array}{l}\text { Modo de recogida } \\
\text { de información }\end{array}$ & Cuestionario realizado en persona o mediante correo electrónico \\
\hline Número de cuestiones & 23 \\
\hline Tipo de cuestiones & $\begin{array}{l}\text { Preguntas abiertas, preguntas cerradas o dicotómicas, preguntas con } \\
\text { respuesta en abanico, preguntas de estimación y preguntas de final } \\
\text { abierto }\end{array}$ \\
\hline $\begin{array}{l}\text { Período de proceso de recogida } \\
\text { de la información }\end{array}$ & Abril-julio de 2010 \\
\hline
\end{tabular}

Tabla 1. Características del cuestionario dirigido a los responsables de Servicios Sociales municipales de la Comunidad de Madrid.

exploratorias a expertos en Servicios Sociales, comunicación pública y marketing de servicios públicos, así como un grupo de discusión conformado por técnicos de Servicios Sociales municipales de la Comunidad de Madrid.

Por su parte, para la disposición de las variables de observación se han incluido preguntas abiertas y cerradas y, dentro de las preguntas cerradas, preguntas dicotómicas y preguntas categóricas de elección múltiple, de respuesta múltiple y de escala. Estas preguntas se han ordenado jerárquicamente en torno a dos grandes dimensiones referidas a:

a) El proceso de elaboración de la difusión de los servicios (organización, objetivos, destinatarios, problemática, recursos, comunicación interna).

b) El empleo de las técnicas de comunicación de que disponen las instituciones públicas para la promoción de los servicios (planificación estratégica de las acciones de comunicación, técnicas utilizadas, emplazamientos, publicidad y aplicación de procesos de marketing).

Para llevar a cabo el análisis, se optó por una metodología estadística simple, basada en métodos descriptivos y en técnicas de estadística inferencial, desvelando relaciones descriptivas y proporcionales.

Así, se han observado en las distintas variables medidas como las medias aritméticas, los modos, las medianas, así como las frecuencias y porcentajes de respuesta. También se ha empleado la prueba de chi cuadrado para desvelar relaciones significativas entre variables, especialmente en variables como el tamaño o el color político de los municipios.

\section{Resultados y discusión}

\subsection{Análisis del cuestionario}

El número de ayuntamientos de la Comunidad de Madrid que finalmente han participado en la investigación ha sido de 20 , un 65 por ciento del universo total de los municipios a quienes se dirigió el cuestionario (31 municipios en 2010), prestando los ayuntamientos participantes estos servicios a un 79 por ciento de los habitantes censados en ese momento en la región. Esta muestra estaba compuesta por municipios de diverso tamaño, regidos por opciones políticas diversas (Partido Popular, Partido Socialista Obrero Español, Izquierda Unida y Partido Independiente de Coslada) (Tabla 2).

\subsubsection{Denominación de las áreas de Servicios Sociales}

Los resultados muestran que la denominación de las áreas de Servicios Sociales difiere prácticamente en la totalidad de los municipios encuestados. Así, encontramos concejalías y departamentos que emplean diversos denominaciones para referirse a la prestación de estos servicios: Departamento de Bienestar Social, Área de Asuntos Sociales e Igualdad, Concejalía de Fa- 


\begin{tabular}{|l|l|l|}
\hline \multicolumn{1}{|c|}{ Tipología } & \multicolumn{1}{|c|}{ N. ${ }^{\circ}$ de habitantes } & \multicolumn{1}{c|}{ Municipios participantes } \\
\hline $\begin{array}{l}\text { Municipios } \\
\text { semiurbanos }\end{array}$ & $\begin{array}{l}\text { Entre 20.001 y 50.000 } \\
\text { habitantes }\end{array}$ & $\begin{array}{l}\text { Ciempozuelos, Mejorada del Campo, Tres Cantos, Boadilla } \\
\text { del Monte, Colmenar Viejo y Villaviciosa de Odón }\end{array}$ \\
\hline $\begin{array}{l}\text { Municipios } \\
\text { urbanos } \\
\text { medianos }\end{array}$ & $\begin{array}{l}\text { Entre 50.001 y 100.000 } \\
\text { habitantes }\end{array}$ & $\begin{array}{l}\text { Collado Villalba, Rivas-Vaciamadrid, Majadahonda, San Se- } \\
\text { bastián de los Reyes, Pozuelo de Alarcón y Coslada }\end{array}$ \\
\hline $\begin{array}{l}\text { Municipios } \\
\text { urbanos } \\
\text { grandes }\end{array}$ & $\begin{array}{l}\text { A partir de 100.001 } \\
\text { habitantes }\end{array}$ & $\begin{array}{l}\text { Alcobendas, Getafe, Alcorcón, Leganés, Fuenlabrada, Alcalá } \\
\text { de Henares, Móstoles y Madrid }\end{array}$ \\
\hline
\end{tabular}

Tabla 2. Municipios participantes en el cuestionario, según tamaño de población.

milia y Asuntos Sociales, etc. Las expresiones más utilizadas para señalar su ámbito de actuación son: Servicios Sociales, Bienestar Social, o Asuntos Sociales (a las que en numerosas ocasiones se les añaden otras expresiones que expresan otros ámbitos de intervención: Familia, Mayores, Inmigración, Igualdad, Mujer, Integración Social o Juventud. En el 30 por ciento de los municipios de la muestra la expresión Servicios Sociales ha desaparecido plenamente (Tabla 3).

2.1.2. Objetivos, orientación y destinatarios principales de la comunicación

Interrogada la ciudadanía sobre ocho posibles objetivos, denominados prioritarios, bajo los que se enmarca la difusión de los Servicios Sociales de sus municipios, la difusión de la existencia de los servicios ha sido el objetivo más veces calificado. Le han seguido la prevención del riesgo social y el fomento del uso de los

\begin{tabular}{|l|c|}
\hline \multicolumn{1}{|c|}{ Denominación del área } & $\begin{array}{c}\text { Número de } \\
\text { respuestas }\end{array}$ \\
\hline $\begin{array}{l}\text { Servicios Sociales y otras } \\
\text { denominaciones }\end{array}$ & 8 \\
\hline Servicios Sociales & 6 \\
\hline Bienestar Social & 2 \\
\hline $\begin{array}{l}\text { Bienestar Social y otras } \\
\text { denominaciones }\end{array}$ & 1 \\
\hline Asuntos Sociales & 1 \\
\hline $\begin{array}{l}\text { Asuntos Sociales y otras } \\
\text { denominaciones }\end{array}$ & 2 \\
\hline
\end{tabular}

Tabla 3. Denominaciones de las áreas de Servicios Sociales. servicios. En menor medida han aparecido nombrados los objetivos de visibilidad de los servicios, mostrar sus beneficios sociales y la sensibilización social.

En cuanto a la orientación de la comunicación, se observa un equilibrio entre los municipios que afirman dirigir sus acciones comunicativas, a partir de un enfoque universal (es decir, dirigido hacia toda la ciudadanía sin distinción) y los que las dirigen hacia sectores específicos y colectivos determinados (enfoque selectivo). Muy pocos municipios combinan ambos enfoques (combinación de enfoques) (Figura 1).

Un enfoque selectivo supone tener como destinatarios principales de las acciones de difusión a colectivos de usuarios ya definidos, y por lo tanto una menor presencia de las acciones de comunicación dirigidas al conjunto general de la ciudadanía.

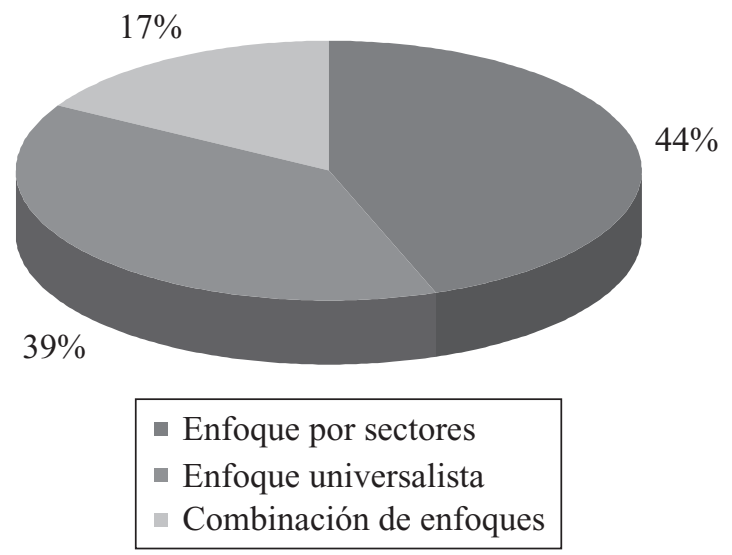

Figura 1. Enfoques de la comunicación de los Servicios Sociales (por ciento). Fuente: Elaboración propia. 
En cuanto a los destinatarios a quienes se dirige la difusión de los servicios, independientemente de los enfoques empleados, el reparto porcentual de destinatarios de la difusión de los servicios muestra que un número mayoritario de municipios elige, entre sus destinatarios prioritarios, a públicos que se ajustan más a lo que podríamos considerar un enfoque por sectores de la comunicación: usuarios directos de los Servicios Sociales, sectores concretos de la población del municipio y público potencial de los servicios.

Estos datos muestran la importancia que el enfoque sectorial tiene en la difusión actual de los Servicios Sociales, independientemente del color o del tamaño de los municipios.

2.1.3. Valor otorgado a la comunicación de los servicios sociales

Al referirnos al valor que se le concede a la comunicación en la estrategia de prestación de los servicios sociales municipales, más de la mitad de las áreas afirman concederle una importancia media, y seis municipios afirman concederle poca importancia; solamente tres de ellos afirman concederle mucha importancia (Tabla 4).

Si el tamaño del municipio no aparece como un factor que influya en este hecho, sí lo es en cambio el color político: hay una relación significativa entre la orientación política de los municipios del estudio y la importancia que se le concede a la comunicación.

A este respecto, una de las responsables encuestadas afirma que «lo poco que se lleva a cabo en materia de comunicación se hace respondiendo más a criterios políticos que a criterios propios de los departamentos». La respon-

\begin{tabular}{|l|c|c|c|}
\hline & $\begin{array}{c}\text { Mucha } \\
\text { importancia }\end{array}$ & $\begin{array}{c}\text { Importancia } \\
\text { media }\end{array}$ & $\begin{array}{c}\text { Poca } \\
\text { importancia }\end{array}$ \\
\hline PP & 3 & 7 & 2 \\
\hline PSOE & & 3 & 3 \\
\hline $\begin{array}{l}\text { Coalición } \\
\text { PSOE-IU }\end{array}$ & & 9 & 7 \\
\hline Total & 3 & & 2 \\
\hline
\end{tabular}

Tabla 4. Importancia concedida a la comunicación en la estrategia de prestación según color político del municipio. sable de otro de los municipios expone otra opinión significativa: «Los servicios sociales nos vendemos solos, por nosotros mismos, al dar servicios, al atender a demandas».

2.1.4. Uso de estrategias de comunicación en la difusión de los servicios

El éxito de un proceso de comunicación se asocia directamente a la implantación y al uso de estrategias comunicativas. Sin embargo, en el caso de los municipios de la investigación, el uso sistemático de estrategias de comunicación es minoritario: solamente dos municipios afirman llevarlo a cabo y seis municipios afirman hacerlo de forma parcial. Los municipios restantes afirman que no llevan a cabo ningún planteamiento estratégico. No obstante, sí se desarrollan de manera general determinadas acciones estratégicas de comunicación en determinados aspectos — planteamiento de objetivos, técnicas, medios, espacios, tiempos o puesta en marcha de evaluaciones - pero obviando una estrategia de conjunto que incluya de manera global todos estos elementos.

\subsubsection{Ubicación de la responsabilidad de la comunicación}

Mediante esta cuestión se ha pretendido determinar dentro de la organización quién decide lo que hay que comunicar y difundir entre la ciudadanía. Es decir, quién construye la imagen de los servicios, dónde se ubica esta responsabilidad. Para ello, se ha intentado dilucidar también hasta qué punto las áreas de Servicios Sociales son independientes para llevar a cabo la difusión de los mismos de las directrices políticas y de qué manera la presencia de departamentos especializados de comunicación de los ayuntamientos influyen en esta difusión. Los datos de la investigación muestran que las áreas o departamentos de Servicios Sociales detentan mayoritariamente el poder de decisión en la elaboración de la comunicación, especialmente desde la dirección de las áreas. Los departamentos de comunicación de los ayuntamientos tienen una presencia, en líneas generales, moderada, así como la dirección municipal, el poder político.

No obstante, se constata que la relación entre el tamaño del municipio y la intervención del poder político es significativa: la presencia del nivel político en la difusión de los servicios 
sociales se intensifica a medida que aumenta el tamaño de los municipios (en municipios semiurbanos y en los grandes municipios). Esto sucede independientemente del color político de los municipios. De cualquier forma las variadas respuestas indican que cada municipio emplea una metodología propia a la hora de difundir sus servicios sociales.

2.1.6. Visibilidad insuficiente de los servicios

Respecto a la visibilidad de los Servicios Sociales, más de la mitad de los encuestados, el 35 por ciento, afirma que sus servicios sufren una situación de baja visibilidad, y el 25 por ciento muy baja, en relación con otros servicios públicos de los municipios.

Tanto en los municipios pequeños como en los municipios grandes los responsables opinan que sus servicios tienen una visibilidad menor de la que consideran que deberían tener. En los municipios medianos, en cambio, los responsables están mayoritariamente satisfechos con su grado de visibilidad, aunque con alguna excepción. La baja visibilidad de los Servicios Sociales públicos en el exterior de los equipamientos municipales es una de las cuestiones que se mencionaron tanto en el grupo de discusión con los técnicos municipales como en las entrevistas exploratorias previas como una de las principales características del modelo de difusión actual.

\subsubsection{Contenidos más difundidos}

Los datos reflejan como unos Servicios Sociales determinados, dirigidos a colectivos específicos, se promocionan más y más frecuentemente que otros. En este sentido, los servicios sociales para personas mayores son los más difundidos. Le siguen los servicios para personas dependientes, los servicios para inmigrantes, y ya en menor medida, los servicios dirigidos a abordar la violencia de género y el maltrato doméstico, los servicios destinados a familias y menores, los servicios de juventud y los servicios para personas con discapacidad. En cambio otros servicios como los de drogodependecias/alcoholismo, los de reinserción social, los de emergencia social o los de autopromoción de los Servicios Sociales han obtenido menores porcentajes de respuesta. Esta variable es interesante porque permite reflexionar sobre la estigmatización de los Servicios Sociales y sobre la asociación de estos servicios a priori universales a determinados colectivos (Figura 2).

En cuanto a la información contenida en las acciones de difusión de los servicios sociales municipales, los contenidos más presentes tienen que ver con la forma de acceso a los mismos y con su utilidad y razón de ser. La difusión de los centros de Servicios Sociales y la titularidad de los servicios están presentes en menor medida y por último, la concienciación o sensibilización ciudadana frente a los proble-

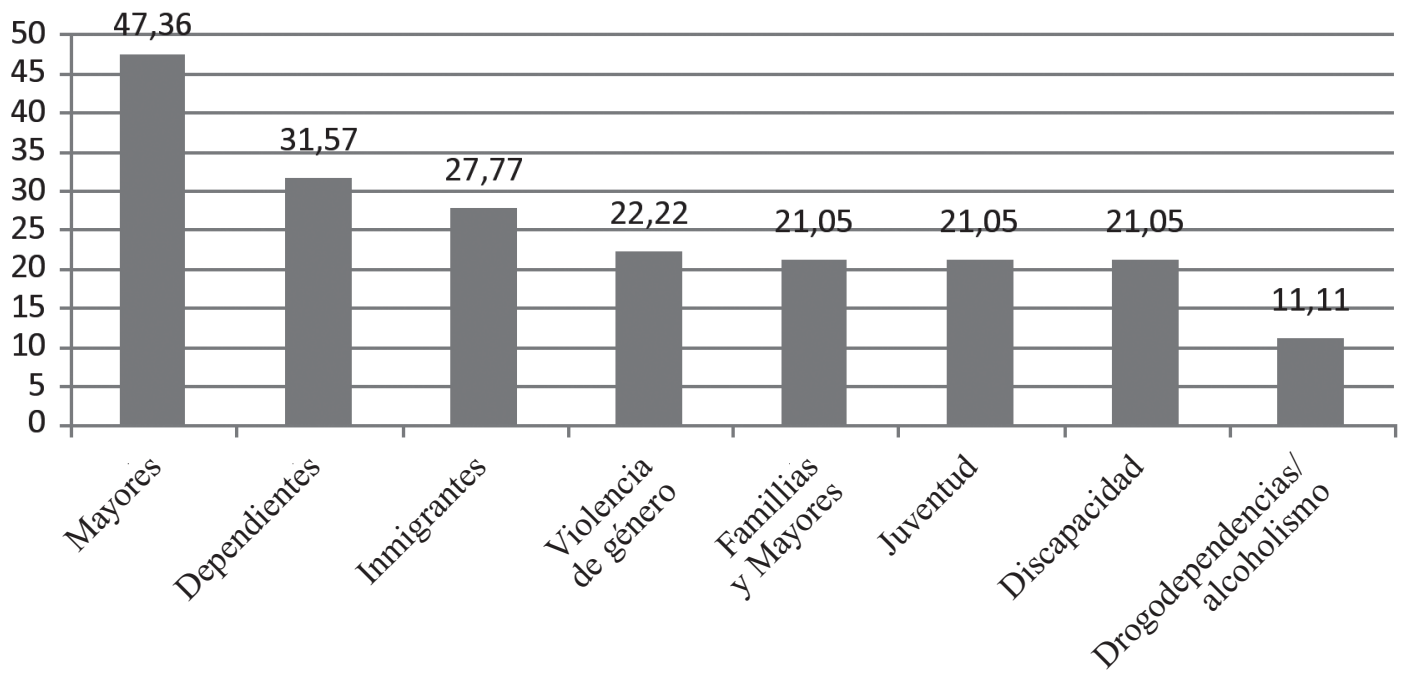

Figura 2. Contenidos más difundidos desde los Servicios Sociales municipales (por ciento acumulados). Fuente: Elaboración propia. 
mas sociales de los municipios o la posibilidad de participación ciudadana tienen una reducida presencia.

2.1.8. Técnicas de comunicación y publicidad

Las técnicas de comunicación y publicidad más empleadas se caracterizan por producir un tipo de comunicación de tipo personal y directa más minoritaria que masiva y en donde la comunicación interpersonal desempeña un papel determinante en la difusión de los servicios: la comunicación boca a oreja (o cara a cara), las entrevistas profesionales o los espacios de encuentro entre profesionales y entre instituciones. Las técnicas que podemos considerar como las más adecuadas para llegar a un público más amplio (tales como Internet-Sitio web, la presencia en medios de comunicación locales o regionales, las publicaciones propias o las campañas sobre determinados servicios) se emplean en un grado menor (Figura 3).

En cuanto a los emplazamientos donde se ubican las acciones de publicidad y difusión de los servicios, es llamativo observar que estas acciones se llevan a cabo generalmente dentro de los propios equipamientos de servicios sociales y en las oficinas o dependencias municipales, y que de manera infrecuente salen de estos espacios, lo que reduce notablemente su posibilidad de impacto.
2.1.9. Aplicación del marketing de servicios públicos

El empleo del marketing de servicios públicos, entendida no solamente como una herramienta publicitaria de los Servicios Sociales, sino como un proceso para la propia elaboración y construcción de los servicios (servucción) a partir del conocimiento de los usuarios, tiene una presencia minoritaria en los servicios sociales municipales de la investigación.

En cambio, se emplean algunas técnicas que hemos asociado a este tipo de marketing ( $\sin$ que sean consideradas como tales por los departamentos): gestión de quejas y sugerencias, acciones de motivación del personal, mejoras de los procesos de interacción, acciones de alta visibilidad, o evaluaciones de la satisfacción de los usuarios.

La cuestión del empleo del marketing de servicios públicos por parte de los Servicios Sociales genera una cierta controversia. Así, en el grupo de discusión con trabajadores se ha identificado un cierto rechazo el asociar la propia expresión marketing con los Servicios Sociales porque se entiende vinculada a ciertos conceptos como mercantilismo, negocio o dinero. No obstante, la gran mayoría de los responsables encuestados ha considerado que el marketing debería formar parte de la estrategia de prestación de los mismos, como se utiliza con otros servicios públicos.

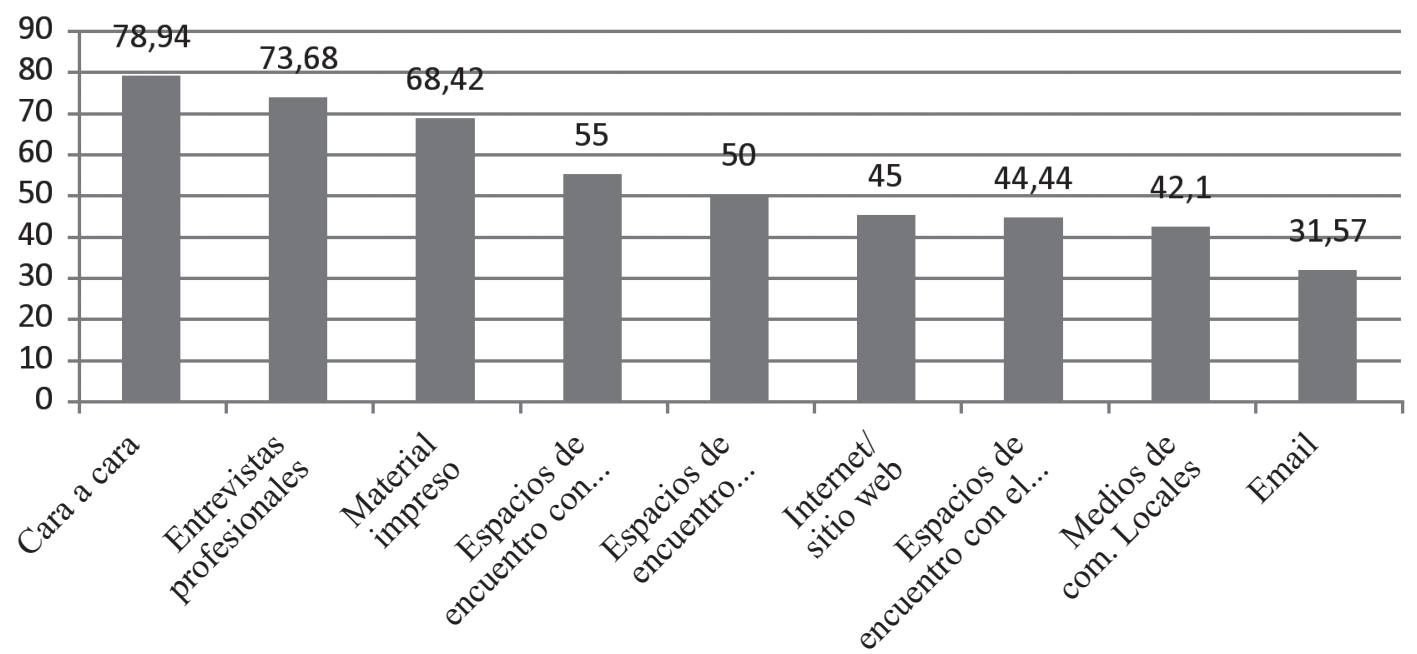

Figura 3. Técnicas de comunicación más utilizadas para difundir los Servicios Sociales municipales (por ciento acumulados).

Fuente: Elaboración propia. 
2.1.10. La imagen de los Servicios Sociales en los medios de comunicación

En cuanto a la percepción que tienen los responsables sobre la imagen que se ofrece de los Servicios Sociales en los medios de comunicación, encontramos un equilibrio entre los responsables que sostienen la existencia de una distorsión de la realidad de los servicios en estos medios y los que no están de acuerdo. La percepción negativa aumenta cuanto mayor es el tamaño del municipio. En cualquier caso, es llamativo observar que persiste de forma significativa (ya que cuenta con la mitad de los participantes en el cuestionario) la opinión de que los medios de comunicación no muestran con exactitud la realidad de los Servicios Sociales y que estos medios ofrecen una imagen distorsionada de los mismos.

2.1.11. Problemática de la difusión de los servicios

Por último, los responsables han señalado una serie de problemas con los que se enfrentan cuando difunden los Servicios Sociales, identificándose mayoritariamente como la principal barrera su estigmatización y señalándose otras veces como la falta de información ciudadana sobre lo que son los Servicios Sociales, la falta de recursos disponibles que padecen las Administraciones locales para poder destinar a la comunicación o el tratamiento inadecuado de la información social por parte de los medios de comunicación (Figura 4).

2.1.12. Influencia del tamaño y del color político de los municipios

Los datos del estudio han mostrado que, en general, no se puede afirmar que haya un tipo de difusión característico de una ideología política u otra. Los Servicios Sociales municipales, independientemente de la ideología política de sus gestores, parecen enfrentarse a las mismas barreras de manera habitual, reflejando inquietudes muy similares en cuanto al cómo comunicarse con la ciudadanía.

Sin embargo, como hemos señalado, se han podido comprobar diversas relaciones significativas entre el color político de los ayuntamientos y ciertos aspectos, como la importancia que se afirma conceder a la difusión de los servicios o el empleo de herramientas para recoger la opinión de los usuarios. Así como con

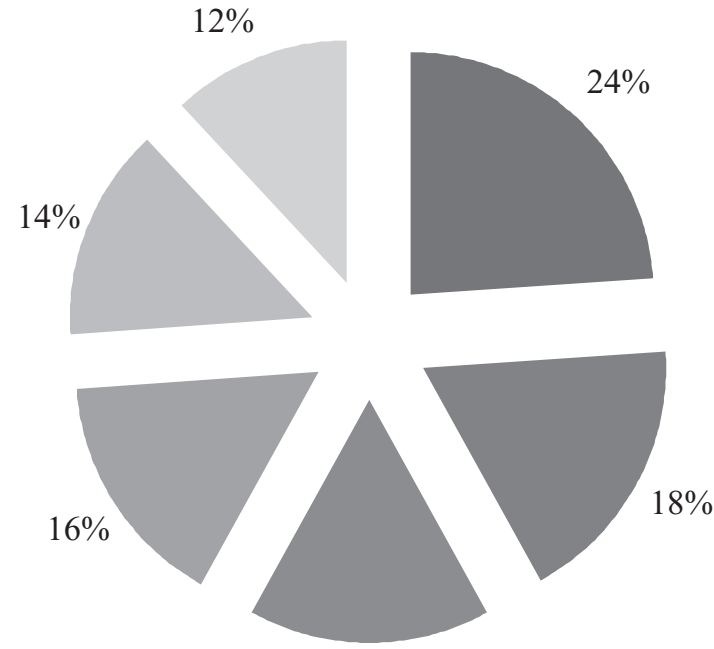

$16 \%$

Estigmatización de los
servicios
Falta de información
ciudadana sobre lo que son
los servicios sociales
Problemas de índole
económica
Tratamiento negativo de los
medios de com.
Descoordinación entre
entidades
Trabas burocráticas

Figura 4. Principales barreras identificadas en la difusión de los Servicios Sociales municipales (por ciento).

Fuente: Elaboración propia.

que la influencia del poder político en la difusión de los servicios es mayor a medida que aumenta el tamaño de los municipios.

En cuanto al tamaño de los municipios, el dato principal que podemos extraer es que la comunicación con la ciudadanía es más difícil en los municipios grandes o muy grandes urbanos, y que parece ser más satisfactoria (en términos de eficacia comunicativa) para los responsables de los municipios medianos.

\subsection{El modelo actual de difusión de los Ser- vicios Sociales municipales}

El conjunto de estos datos nos permite emitir una serie de valoraciones sobre cómo es la co- 
municación de los Servicios Sociales de Atención primaria en el territorio determinado y en el momento de la investigación:

- Un modo confuso de referirse a los Servicios Sociales (empleándose diversas denominaciones para ello e incluso haciendo desaparecer el término servicios sociales)

- Una tendencia a realizar un enfoque cada vez más selectivo de la comunicación de los servicios, lo que aumenta las posibilidades de recepción de la información por parte de los colectivos definidos a los que se dirige, pero también limita las posibilidades de conocimiento de otros sectores de la ciudadanía que pueden ser objeto de la atención social y no tienen porqué estar incluidos dentro de estos colectivos.

- Un uso minoritario de estrategias globales o planes estratégicos de comunicación para difundir de manera general los servicios sociales en sus municipios.

- Una diversidad en la forma de organizar, en los distintos departamentos de Servicios Sociales, los procesos de difusión y de promoción de los mismos. Diversidad acompañada de una influencia notable de otras áreas ajenas a estos departamentos, especialmente la influencia del nivel político es mayor a medida que aumenta el tamaño del municipio.

- Una visibilidad reducida de los servicios en los municipios en relación con otros servicios públicos, disponiendo las áreas de Servicios Sociales de escasos recursos para emplear en comunicación y publicidad.

- Una difusión desigual de los Servicios Sociales: unos servicios determinados dirigidos a unos colectivos definidos se difunden más que otros: especialmente los servicios para personas mayores. Este hecho puede suponer que la atención social, que tiene un carácter universal, se relacione con determinados colectivos a ojos de la ciudadanía.

- Un predomino del uso de técnicas de comunicación de carácter personal, directo y minoritario, en detrimento del uso de técnicas de comunicación con capacidad de alcanzar a un número mayor de ciudadanos.

El empleo del marketing de servicios públicos en los Servicios Sociales municipales es prácticamente inexistente.

\section{Conclusiones}

A tenor de los resultados del trabajo, puede considerarse que la estructura actual del sistema público de Servicios Sociales impide que se esté promocionando de manera homogénea, integral y universal. Lo que a su vez supone que se está difundiendo al conjunto de la ciudadanía española de forma desigual, limitada, selectiva, heterogénea y confusa. Al menos en lo referido a los servicios sociales de Atención Primaria, puerta de entrada al sistema.

El estudio refleja además una minusvaloración del factor comunicativo en las instituciones públicas encargadas de la prestación de los servicios sociales municipales, reflejada en una escasa inversión en publicidad y comunicación, debido probablemente a una escasez de recursos económicos. Una cuestión que, sin duda, está ligada a la reducción de la generosidad del sistema, cuando no de su universalidad.

La persistencia de las características de este modelo de difusión genera una situación en la que, sin duda, se dificulta que pueda elevarse el grado de conocimiento del sistema por parte de la ciudadanía, lo que no excluye que puedan existir otros factores que afecten a este conocimiento. «Si un ciudadano no tiene información de una oferta de servicios, a pesar de que objetivamente dicha persona pueda reunir todos los (demás) requisitos establecidos para disfrutar del servicio, es imposible, por ignorancia, que los reciba) (Charroalde, 1996, p. 39).

El desconocimiento tiene además dos consecuencias: genera confusión y preserva la estigmatización histórica existente en determinados sectores respecto a la percepción general del conjunto del sistema. Desconocimiento, confusión y estigmatización son tres elementos que determinan notablemente el acceso ciudadano a los servicios sociales públicos. Lo que no se conoce, no se puede utilizar ni se puede exigir.

Así, nuestra conclusión principal es que el sistema no se está difundiendo de manera universal. Pese a que la recogida de información se realizó en 2010, la situación con respecto a la difusión del sistema no ha experimentado ningún cambio significativo ya que la organización del sistema tampoco lo ha hecho, a la espera de la probable reforma de la Administración local. Así pues, estas conclusiones si- 
guen siendo igual de válidas hoy que ayer desde la perspectiva de observación a partir de las cuáles han sido elaboradas.

Si bien la muestra se ha circunscrito a la Comunidad de Madrid, la legislación española de Servicios Sociales y la organización administrativa que deriva de ella hacen suponer que esta manera de difundir los Servicios Sociales está teniendo lugar en todo el territorio nacional. En España no existe una ley general de Servicios Sociales que implique al conjunto del territorio, como sí existe en torno a la educación o a la sanidad. Por tanto, no hay una política concreta, común y estratégica para difundir el sistema público de los mismos.

Todo ello nos lleva a reflexionar, en el ámbito de la comunicación, sobre la necesidad de una intervención de un nivel administrativo superior en este sentido. Sin embargo, la reflexión nos lleva a plantear dos grandes obstáculos para poder hacerlo: la organización administrati- vo-territorial de los Servicios Sociales públicos y la financiación del sistema ¿Sería posible implementar una política global de difusión y promoción del sistema desde el Estado o las comunidades autónomas? Sería necesario si se pretendiese aumentar el conocimiento global de los Servicios Sociales, pero esto demandaría una disposición de las administraciones públicas concernidas que, hasta la fecha, no es posible alcanzar dada la estructura actual del sistema español. La posible reforma de la Administración local podría suponer una oportunidad en esta perspectiva.

Por otra parte (y quizá aquí encontremos el nudo de la cuestión), en un contexto como el actual donde, pese al aumento de la exclusión social y de la precariedad en la sociedad española, la sostenibilidad económica del Estado de bienestar español está puesta en cuestión más que nunca ¿es conveniente difundir más y mejor los Servicios Sociales públicos?

\section{Referencias bibliográficas}

Bueno Abad, J. R. y Pérez Cosín, J. V. (2000). Percepciones de los servicios sociales y representaciones de los trabajadores sociales. Cuadernos de Trabajo Social, 13, 53-74.

Charroalde, J. (1996). Información y orientación en los Centros de servicios sociales. Cuadernos de Trabajo Social, 9, 38-41.

Fantova, F. (2003). Perspectivas en gestión de servicios sociales. Puresoc. Publicaciones y recursos de sociología y de áreas afines. Universidad Pública de Navarra. Recuperado de:http://www.unavarra.es/puresoc/pdfs/c_lecciones/LM-Fantova.pdf

García Herrero, G. y Ramírez Navarro, J. M. (2001). Imagen y Comunicación en temas sociales. Zaragoza: Editorial Certeza y Consejo General de Diplomados en Trabajo Social.

Grande Esteban, I. (1993). Marketing estratégico para la Tercera Edad. Madrid: ESIC.

Grande Esteban, I. (1996). Marketing de los servicios. Madrid: Síntesis.

Grande Esteban, I. (2002). Marketing de los servicios sociales. Madrid: Síntesis.

Guillén, E. y Vilà, A. (2007). Los cambios legislativos recientes en materia de servicios sociales. En D. Casado y F. Fantova (coords.). Perfeccionamiento de los servicios sociales. Informe con ocasión de la Ley sobre autonomía y dependencia. Madrid: Fundación Foessa, Cáritas Española y Caja Madrid Obra Social.

Informe EDIS. (2000). Las condiciones de vida de la población pobre desde una perspectiva territorial. Madrid: Fundación FOESSA.

Kahn, A. J. y Kamerman, S. B. (1987). Los servicios sociales desde una perspectiva internacional: el sexto sistema de protección social. Madrid: Siglo XXI.

Las Heras, M. P. (2002). El sistema público de servicios sociales. Ponencia presentada en el seminario El reto de la universalidad de derechos en servicios sociales. (Documento de trabajo). Fundación Pablo Iglesias, Madrid.

Marcuello, Ch. y Gutiérrez Resa, A. (2008). Servicios sociales: modelos y perspectivas. Madrid: Ed. Ediasa.

Muñoz Luque, J. (2009). Servicios Sociales de atención primaria en España. En V. Navarro. (dir.), La situación social de España, vol. III Biblioteca Nueva; Programa de Políticas Públicas y Sociales de la Universidad Pompeu Fabra,. Madrid: Fundación Francisco Largo Caballero. 
Perdomo Molina, S. (septiembre-diciembre, 2009). Servicios Sociales. Planteamientos de identidad y necesidad de un sistema. Revista Trabajo Social Hoy, 58, 81-110.

Puig i Picart, T. (2004). Marketing de servicios públicos para administraciones públicas con los ciudadanos. En red, claves y entusiastas. Sevilla: Dirección General de Administración Local de la Junta de Andalucía.

Subirats Humet, J., Adelantado Gimeno, J., Fernández Prat, M., et al (2007). Los Servicios Sociales de Atención Primaria ante el cambio social, Madrid: Ministerio de Trabajo y Asuntos Sociales.

Warin, P. (2010) Le non-recours: définition et typologies. (Documento de trabajo). Grenoble: Odenore (Observatoire des non- recours aux droits et services) et Maison des Sciences de 1'Homme-Alpes.. 